\title{
Correction to: Project honeybee: Clinical applications for wearable biosensors
}

\author{
Leland Hartwell ${ }^{1}$ - Heather M. Ross ${ }^{2,3}$ • Jeffrey T. La Belle ${ }^{4,5}$ \\ Published online: 12 September 2020 \\ (C) Springer Science+Business Media, LLC, part of Springer Nature 2019
}

\section{Correction to: Biomedical Microdevices (2019) 21: 37 https://doi.org/10.1007/s10544-019-0392-y}

The original version of this article unfortunately contained a mistake. The two sentences in the Abstract section 'Please provide an abstract of 150 to 250 words. The abstract should not contain any undefined abbreviations or unspecified references.' should be removed. The corrected Abstract is given below.
The online version of the original article can be found at https://oi.org/ 10.1007/s10544-019-0392-y

Jeffrey T. La Belle

JEFFREY.LABELLE@asu.edu

1 Biodesign Pathfinder Center, Biodesign Institute at Arizona State University, Tempe, AZ 85281, USA

2 School for the Future of Innovation in Society, Tempe, AZ 85287, USA

3 College of Nursing and Health Innovation, Tempe, AZ 85004, USA

4 School of Biological and Health Systems Engineering, Arizona State University, Tempe, AZ 85287, USA

5 School of Medicine, Mayo Clinic Arizona, Scottsdale, AZ 85259, USA

\begin{abstract}
The Project Honeybee Observational Clinical Trials were 12-month studies designed to validate the use of commercially available ambulatory medical devices costing $\$ 50-\$ 300$ for clinical applications. Each trial had a patient population of about 15-30 subjects with a broad range of disease types including heart failure, diabetes, sepsis, and Parkinson's disease. Over 30 supported proposals were funded in the 4-year period, as well as the creation of a database of all commercially available devices. Each year a call for proposals was published within ASU and Mayo Clinic Arizona. Proposals were selected for funding by a committee of ASU faculty from engineering, nursing, and exercise physiology departments. The progress of each research trial was monitored through monthly colloquia with the nursing, biomedical engineering, computer science, and nutrition graduate research assistants, to discuss the challenges and opportunities arising with each research trial. PIs were required to report on study progress 6 months into the trial period and 3 months following the conclusion of the 12-month project. The project was very successful in meeting our goals of testing consumer wearable devices on patients for a variety of conditions across a variety of clinical settings in the greater Phoenix community. The following clinical sites participated in one or more of these clinical trials: Adelante Healthcare, Arizona Arrhythmia Consultants, Arizona Cardiology Group, Banner University Medical Center, Barrow Neurological Institute, Honor Health, Mayo Clinic, and St Joseph's Hospital. A total of 12 ASU faculty and 39 clinicians participated.
\end{abstract}

Publisher's note Springer Nature remains neutral with regard to jurisdictional claims in published maps and institutional affiliations. 